\title{
Plugs in Viscometric Flows of Simple Semi-liquids
}

\author{
Walter NOLL and Brian SEGUIN ${ }^{\dagger}$ \\ Department of Mathematical Sciences, Carnegie Mellon University \\ 5000 Forbes Ave., Pittsburgh, PA, 15213-1662, USA \\ (Received : June 27, 2008)
}

In 1966, Coleman, Markovitz and Noll published a book entitled Viscometric Flows of Non-Newtonian Fluids. The analysis presented there is based on the concept of an incompressible simple fluid (simple liquid), which Walter Noll had introduced in 1958. About 5 years ago he introduced the concept of a simple semi-liquid. It turns out that the analysis given in the book mentioned above can be extended to simple semi-liquids. The behavior of simple liquids in viscometric flows is characterized by three functions: the shear-stress function and two normal stress functions. This remains true for simple semi-liquids, but now the limits of these functions as the rate of shear goes to zero need not be zero but may approach critical non-zero values. This fact allows the existence of plugs. This paper gives explicit procedures for determining the size of plugs for the entire variety of viscometric flows analyzed in the book mentioned above.

Key Words: Viscometric flows / Plugs / Non-Newtonian fluids / Simple semi-liquids

\section{INTRODUCTION}

\subsection{Simple Semi-liquids}

About 50 years ago, in 1958, one of us (W.N.) introduced the concept of a simple fluid (see 1)). It is based primarily on two ideas:

1) The present stress in a material element is determined by the history of its deformation.

2) The symmetry group of the element is the full unimodular group.

This concept provides a general framework which includes almost all previously proposed mathematical models for Non-Newtonian fluids as special cases and also, of course, the classical theory of Navier-Stokes fluids. It turned out that only this general concept, and no special mathematical models, were needed to analyze the behavior of simple liquids (incompressible simple fluids) in 2). This analysis was presented, in detail, in Chapter E of 2), published first in 1965, and in 3), published in 1966.

The theory of simple liquids could not describe the mechanical behavior of many of the materials studied by rheologists, for example the phenomena described by what

$\dagger$ To whom correspondence should be addressed.

E-mail: bseguin@andrew.cmu.edu they call "Bingham materials". Also, it did not include Ericksen's Theory of Anisotropic Fluids as a special case. Aware of these shortcomings, W.N. introduced, in 1972, what he called the New Theory of Simple Materials (see 4)). It is based primarily on the following ideas:

a) With each material element one associates a State Space. A state, i.e., an element of this space, determines the present configuration of the element and the present stress in it.

b) Given the initial state of a material element and subjecting it to an arbitrary deformation process, the final state is determined.

He proved that the old theory of simple materials is a special case of the new one. However, the new theory gives a framework for plastic behavior of materials that the old one does not. The new theory has not seen many applications for about 30 years. About 5 years ago, he took the topic up again and introduced the concept of a simple semi-fluid, which is a simple material, in the new sense, whose symmetry group is the full unimodular group (see part 4 of 5), entitled The Theory of Simple Semi-Liquids, a Conceptual Framework for Rheology). It turns out that only this general concept, and no special mathematical models, are needed to analyze the behavior of simple semi-liquids (incompressible simple semifluids) in viscometric flows. The analysis of viscometric flows, 
described in 2) and 3) mentioned above, can be extended to those semi-liquids. The new theory, however, allows the existence of plugs (regions of constant velocity) in such flows, while the old theory does not.

The new theory may give a mathematical framework for describing some of the behavior of such familiar materials as toothpaste, ketchup, mustard, and sour cream. A special case of the new theory is what W.N. called Nematic Semi-Liquids (see part 5 of 5)), which in turn includes Ericksen's Theory of Anisotropic Fluids (see Sects. 127-129 of 2)) as a special case.

The behavior of simple liquids in viscometric flows is characterized by three functions: the shear stress function and two normal stress functions. The limit of these functions as the rate of shear goes to zero is zero. The behavior of simple semi-liquids in viscometric flows is characterized again by these three functions, but their limits as the rate of shear goes to zero approaches critical values that need not be zero. It is the critical value of the shear stress that determines the size of possible plugs. The purpose of this paper is to give explicit procedures for determining the size of plugs for the entire variety of viscometric flows described in 3$){ }^{1}$

The critical values of the normal stress functions may lead to a modification of the analysis of the effects of normal stresses, in particular the climbing in a steady Couette flow and swelling at exit from a pipe in Poiseuille flow. Such modification will have to be considered in later papers.

\subsection{Caution}

Viscometric flows may not be achievable in some simple semi-liquids. Certain limiting conditions must be satisfied for this to be the case. (See Assumptions I, II, III in part 4 of 5).) The mathematical treatment given below presume steady motions and idealized boundaries that are infinite or free. An infinite time may be required to establish a steady flow. Also, the critical values of the stresses mentioned above need not be the same as yield stresses. It may happen that larger stresses must be applied to initiate a viscometric flow. The critical stresses determine at what stage a viscometric flow stops when it is gradually slowed down.

\subsection{Experimental Methods and Results}

The statements made in Sect. 23 of 3) apply here, too. Flows that can be achieved in the laboratory can be described only approximately by the results given here. The experimenter must consider, for example "end effects" due to the finite

\footnotetext{
1 For Poiseuille flow and Couette flow, such plugs were discussed already at the end of Sect.6 of part 4 of 5). The analysis of some other flows, especially in Sect. 4.3, depends on some crucial insights by Brian Seguin.
}

dimensions of the apparatus. The results below that are most likely to be of interest to experimenters are given in Sections 3(b), 5, 7, 8, and 9.

As in 3) we always assume that the material adheres to the bounding surfaces, i.e., that there is no slip. Whether such slip occurs depends not only on the nature of the material, but also on the stickiness of the bounding surfaces. Hence the analysis of such slips is likely to be very complicated and beyond the scope of this paper. However, we believe that, in some cases, what looks like a slip is merely the fact that that most of the motion is in the plug. For example, in Poiseuille flow, the plug may cover $99 \%$ of the cross section of the tube and the actual flow is confined to the remaining $1 \%$. It is then still a flow, not a slip. We are convinced that this often happens for materials such as toothpaste and ketchup.

We are aware that there are many papers on plugs in the literature of rheology; 6) is an example. However, it seems that most if not all of the theoretical treatments in the past assume that the shear stress function conforms to a special model, such as the models of Bingham, Herschel-Bulkley, or whatever. These models do not refer to a complete constitutive laws but only to a particular forms of the rate of shear function. The whole point of this paper is to show that special models are not needed to analyze plugs. Shear-stress functions should be determined experimentally by using, for example, the formula (8) in Sect. 3.2.1 or the methods described at the end of Sects. 4.2.1 or 4.4.1 below. It is a bad idea to assume, a priori, that these functions conform to any special model.

Experimenters should be encouraged to produce a catalogue of graphs of shear-stress functions for a variety of materials. This catalogue can then be used to predict the behavior of these materials in any viscometric flow. We hope that our paper may induce some experimenters to reconcile our results with experimental data for semi-liquids in which plugs can occur, just as Hershel Markovitz reconciled the results by Coleman and Noll for liquids in which plugs cannot occur, as described in 3).

To make things easier for an experimenter, we put the final results for each kind of flow at the end of each relevant section. We also include the special form of these results for Bingham materials, partly just as an example and partly to show that they coincide with results already in the literature on rheology.

\subsection{Mathematical Background}

Unfortunately, most physicists are still stuck with an outdated mathematical infrastructure, using variables, constants, and parameters rather than sets and mappings. (See the essay The Conceptual Infrastructure of Mathematics 
on the website www.math.cmu.edu wn0g/noll.) We believe that the new mathematical infrastructure will prevail even in physics, but it may take another 50 years.

However, in this paper, only very little of this new infrastructure is needed, and it is explained in Sect. 2.1 below. The experimental rheologist need not be familiar with the general theory of simple semi-liquids.

\section{PRELIMINARIES}

\subsection{Mathematical Infrastructure}

We use the terminology and notation of contemporary mathematics as described, for example, in Sect. 0 of 7). Here are some particulars:

Writing $f: D \rightarrow C$ means that $f$ is a mapping described by the set $D$, the domain of $f$, the set $C$, the codomain of $f$, and a procedure that associates with each $x \in D$ a value $f(x) \in C$. We say that $f$ is injective if, for every $y \in C$, there is at most one $x \in D$ such that $f(x)=y$. We say that $f$ is surjective if, for every $y \in C$, there is at least one $x \in D$ such that $f(x)=y$. The mapping $f$ is both injective and surjective if and only if, for every $y \in D$, there is exactly one $x \in D$ such that $f(x)=y$. In that case, one can define the inverse $f^{\leftarrow}: C \rightarrow D$ by the procedure which associates with each $y \in C$ the only $x:=f^{\leftarrow}$ $(y) \in D$ which satisfies $f(x)=y$.

The range of a given mapping $f: D \rightarrow C$ is the set Rng $f:=\{f(x) \mid x \in D\}$ of all values of $f$. The codomain must be given a priori, but determining the range can be a difficult problem. The mapping is surjective if and only if $\operatorname{Rng} f=C$. The adjustment $\left.f\right|^{\text {Rng }}$ is obtained from $f$ simply by changing the codomain to the range. Thus, if $f$ is injective, then $\left.f\right|^{\text {Rng }}$ is invertible.

The set of all real numbers is denoted by $\mathbb{R}$, the set of all positive reals, including 0 , is denoted by $\mathbb{P}$, and the set of all strictly (i.e. non-zero) positive reals is denoted by $\mathbb{P}^{\times 2}$.

The term function is usually used for mappings whose codomain is $\mathbb{R}$ or a subset thereof.

Let $f: D \rightarrow C$ be a function whose domain and codomain are subsets of $\mathbb{R}$. We say that $f$ is isotone if , for all $x, x^{\prime} \in D$, $x<x^{\prime}$ implies $f(x) \leq f\left(x^{\prime}\right)$. We say that $f$ is strictly isotone if, for all $x, x^{\prime} \in D, x<x^{\prime}$ implies $f(x)<f\left(x^{\prime}\right)$. The terms antitone and strictly antitone are defined similarly. If a function is strictly isotone or strictly antitone, it is injective.

If $f$ is a difierentiable function we denote its derivative by $f^{\bullet}$, honoring Newton. ${ }^{3}$

2 It is useful to consider zero to be a number that is both positive and negative.

3 The more common notation $f^{\prime}$ may clash with the use of the prime as a mere distinction mark.

\subsection{The Rate of Shear Function}

The determining material function for viscometric flows of simple liquids or semi-liquids is the shear stress function $\tau$ : $\mathbb{P} \rightarrow \mathbb{P}$. We assume that $\tau$ is strictly isotone and that its limit at $\infty$ is $\infty$. Its value at zero, $\tau_{o}:=\tau(0)$, is the critical shear stress. For simple liquids we have $\tau_{o}=0$. For Navier-Stokes liquids the graph of $\tau$ is a straight line whose slope is the viscosity.

For the rest of the paper we will consider a simple semiliquid for which critical shear stress $\tau_{o}$ is strictly positive. If $\tau$ is of the form

$$
\tau(\kappa)=\tau_{o}+\eta_{o} \kappa \quad \text { for all } \quad \kappa \in \mathbb{P}^{\times}
$$

then it is called a Bingham material. For small values of $\kappa$ the equation (1) can serve as a good approximation even for a general simple liquid when $\tau$ is continuously difierentiable and we put $\eta_{o}:=\tau^{\bullet}(0)$, which may be called the initial viscosity.

All the considerations of Chapters III and IV of 3) can be applied here, provided they are modified to account for the possibility that $\tau_{o}$ need not be zero.

Our assumptions on $\tau$ insure that Rng $\tau=\tau_{o}+\mathbb{P}$ and that the adjustment $\left.\tau\right|^{\mathrm{Rng}}$ is invertible. The rate of shear function $\lambda: \mathbb{P}$ $\rightarrow \mathbb{P}$ introduced in Sect. 11 of 3 ) must be given the following new definition (see (6.6) and (6.7) of part 4 of 5)):

$$
\begin{aligned}
& \lambda(S):=\left(\left.\tau\right|^{\mathrm{Rng}}\right)^{\leftarrow}(S) \text { if } S \in \tau_{o}+\mathbb{P}^{\times}, \\
& \lambda(S):=0 \text { if } S \in\left[0, \tau_{o}\right],
\end{aligned}
$$

so that

$$
\lambda(\tau(\kappa))=\kappa \quad \text { for all } \kappa \in \mathbb{P} .
$$

It is this function that must be used when dealing with semiliquids. In the case of a Bingham material (2) reduces to

$$
\begin{aligned}
& \lambda(S)=\frac{1}{\eta_{o}}\left(S-\tau_{o}\right) \text { if } S \in \tau_{o}+\mathbb{P}^{\times}, \\
& \lambda(S)=0 \text { if } S \in\left[0, \tau_{o}\right] .
\end{aligned}
$$

The graph of $\lambda$ is illustrated in Fig. 1, with the straight line corresponding to a Bingham material.

In the remainder of this paper, we will make use of the formulas from Sects. 9, 14-19, 21 and 22 of 3), which remain valid when $\lambda$ is given by (2).

\section{FLOWS WITHOUT PLUGS}

\subsection{Simple Shearing}

Consider the situation where the simple semi-liquid is contained between two parallel infinite plates. Choose a Cartesian coordinate system $(x, y, z)$ so that the lower plate is at $x=0$ and the upper plate is at $x=d$. Let a force $F$ per unit 
area be applied to the top plate in the positive $y$-direction so that $T^{<x y>}(d)=F$. Also assume that the sem-liquid adheres to both plates.

In this situation the velocity field is assumed to have the form

$$
v^{<x>}=0, \quad v^{<y>}(x)=\kappa x, \quad v^{<z>}=0,
$$

where $\kappa$ satisfies $\tau(\kappa)=T^{<x y>}=F$ (see (111.6) in 2)). In terms of the rate of shear function $\lambda$ defined by (2), this means that $v^{\langle y\rangle}=\lambda(F) x$. Hence, using (2), we obtain $v^{\langle y\rangle}=0$ when $F \leq \tau_{o}$ and also the following:

\subsubsection{Results}

There is no flow if $F \leq \tau_{o}$, and if $F>\tau_{o}$ then there is flow and $v^{\langle y\rangle}(x)=\lambda(F) x$.

\subsection{Cone and Plate Flow}

Consider the situation where the simple semi-liquid is contained between a rigid cone rotating about its axis with angular velocity $\Omega_{1}$ and a rigid disk of radius $R$ rotating about the same axis with angular velocity $\Omega_{0}$. Define a spherical coordinate system such that the axis of the cone is the polar axis, the rigid disk is in the plane determined by $z=0$ and the angle between the cone and the disk is $\alpha$. We assume that the velocity field has the components

$$
v^{\theta}=0, \quad v^{\phi}=\omega(r), \quad v^{r}=0
$$

and that the boundary conditions

$$
\omega(\pi / 2)=\Omega_{0}, \quad \omega(\pi / 2-\alpha)=\Omega_{1}
$$

are satisfied. It is known that the dynamical equations can not be solved exactly under these conditions (see 3) p. 51). However, if one neglects inertia and assumes the angle $\alpha$ is small, in the sense that $\cos \alpha \approx 0$ and $\sin \alpha \approx 1$, then most of the results of Sect. 21 of 3) apply.

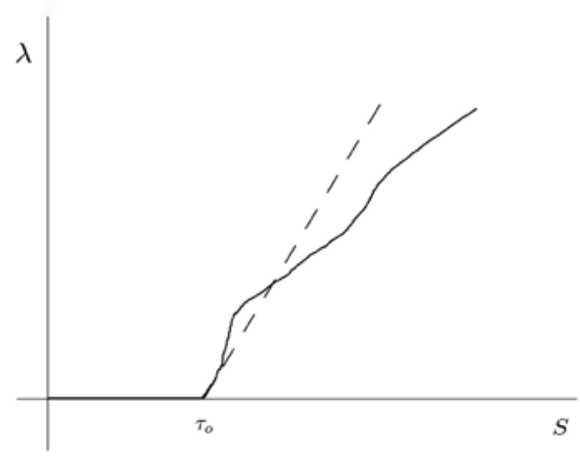

Let $M$ be torque necessary to make the cone turn relative to the plate. Define

$$
M_{o}:=\frac{2 \pi}{3} R^{3} \tau_{o}
$$

It is not hard to arrive at the following:

\subsubsection{Results}

If $M \leq M_{o}$ the material doesn't move relative to the cone and plate and hence $\Omega_{1}=\Omega_{0}$.

If $M>M_{o}$ then the material moves and the torque that must be applied to produce the angular velocity difierence $\Omega_{1}-\Omega_{0}$ is given by the the formula

$$
M=\frac{2 \pi}{3} R^{3} \tau\left(\frac{\Omega_{1}-\Omega_{0}}{\alpha}\right) .
$$

\subsection{Torsional Flow}

We consider a flow that takes place in a cylindrical region bounded by two parallel circular plates, each with radius $R$, separated by a distance $d$. We define a cylindrical coordinate system such that the bottom plate is at $z=0$, the top plate at $z=d$ and the polar axis passes through the centers of the disks.

Torsional flow between these plates is then assumed to have a velocity field whose components are

$$
v^{z}=0, \quad v^{\theta}=\omega(z), \quad v^{r}=0
$$

and which satisfies the boundary conditions

$$
\omega(0)=\Omega_{0}, \quad \omega(d)=\Omega_{1} .
$$

Most of the results of Sect. 22 of 3) apply and so one obtains, by neglecting inertial forces, that

$$
\omega(z)=c z+\Omega_{0} \quad \text { and } \quad \kappa=r c,
$$

where $c$ is a constant.

Let $M$ be the torque applied to the plates designed to make them turn relative to each other and define $M_{o}$ as in (7). It is not hard to arrive at the following:

\subsubsection{Results}

If $M \leq M_{o}$ then $\Omega_{0}=\Omega_{1}$ and the material does not move relative to the plates.

If $M>M_{o}$ the material moves and its velocity is given by equation (22.11) of 3). The torque that must be applied to produce the angular velocity difierence $\Omega_{1}-\Omega_{0}$ is given by the the formula

$$
M=2 \pi \int_{0}^{R} r^{2} \tau\left(\frac{\Omega_{1}-\Omega_{0}}{d} r\right) d r
$$

Fig. 1. Rate of shear function. 


\section{FLOWS WITH PLUGS}

\subsection{Channel Flow}

Consider the steady flow of a column of height $h$ of simple semi-liquid between two infinite parallel plates. We choose a Cartesian coordinate system so that the two infinite plates are perpendicular to the $x$-axis and that the flow is in the direction of the z-axis. Arrange the coordinate system so that one plate is at $x=d$ and the other one is at $x=-d$ and that the column is bounded by $y=0$ and $y=h$. Assume that the fluid adheres to the two plates.

Given this information one can solve for the velocity profile. One obtains

$$
v^{<x>}=0, \quad v^{<y>}=v(x)=\int_{x}^{d} \lambda(f \xi) d \xi, \quad v^{<z>}=0,
$$

where $f$ is the specific driving force, i.e., the applied force per unit volume in the z-direction. (See Sect. 111 in 2)).

Using (2) one can see that the integrand for $v(x)$ in (13) will be zero if $|x| \leq \frac{\tau_{o}}{f}$. Hence we have the following:

\subsubsection{Results}

There is no flow if $f \leq \frac{\tau_{o}}{d}$.

Assume that $f>\frac{\tau_{o}}{d}$. Between the planes defined by

$$
x_{1}:=\frac{\tau_{o}}{f} \text { and } x_{2}:=-\frac{\tau_{o}}{f}
$$

the material will have the constant velocity

$$
v_{p}:=\int_{x_{1}}^{d} \lambda(f \xi) d \xi
$$

Outside of this plug the velocity field is given by

$$
v(x)=v(-x)=\int_{x}^{d} \lambda(f \xi) d \xi \quad \text { for all } \quad x \in\left[x_{1}, d\right]
$$

Let $Q: \mathbb{P}^{\times} \rightarrow \mathbb{P}$ describe the volume discharge, i.e., the volume of material passing through the channel per unit time, as a function of the driving force that produces the flow. The formula (14.22) of 3) reduces to

$$
Q(f)=\frac{2 h}{f^{2}} \int_{0}^{d f} S \lambda(S) d S
$$

\subsubsection{Bingham materials}

In this case, (17) becomes

$$
Q(f)=\frac{h}{3 \eta_{o} f^{2}}\left(2(d f)^{3}-3 \tau_{o}(d f)^{2}+\tau_{o}^{3}\right) .
$$

\subsection{Poiseuille Flow}

Consider a steady flow through a infinite fixed cylindrical tube of radius $R$ produced by a driving force in the axial direction. Choose a cylindrical coordinate system such that the $z$-axis coincides with the axis of the tube. Finally, choose the positive $z$-direction so that the driving force is positive. Assuming that the material adheres to the tube, the results of Sect. 19 of 3) hold and so the velocity profile is of the form

$$
v^{r}=0, \quad v^{z}=u(r), \quad v^{\theta}=0
$$

with

$$
u(r)=\int_{r}^{R} \lambda(\xi f / 2) d \xi
$$

where $u:[0, R] \rightarrow \mathbb{P}$ describes the axial velocity of the flow as a function of the distance from the axis of the tube and $f$ is the specific driving force, i.e., the driving force per unit volume.

Define

$$
f_{o}:=\frac{2 \tau_{o}}{R} \quad \text { and } \quad P:=\frac{2 \tau_{o}}{f} .
$$

There are two possible situations:

\subsubsection{Result}

If $f \leq f_{o}$, and hence $P \geq R$, then $u(r)=0$ for all $r \in[0, R]$ and hence the material doesn't move.

If $f>f_{o}$, and hence $P<R$, then the integrand in (20) is 0 for $r \in[0, P]$ and this interval describes the plug, which is a cylindrical region of radius $P$ moving like a rigid body. The velocity $v_{p}$ of the plug is given by

$$
v_{p}=\int_{P}^{R} \lambda(\xi f / 2) d \xi
$$

Let $Q: f_{0}+\mathbb{P}^{\times} \rightarrow \mathbb{P}$ describe the volume discharge. It follows from (19.9) of 3) and (2) that

$$
\begin{aligned}
& Q(f)=\pi \int_{P}^{R} r^{2} \lambda\left(\frac{r f}{2}\right) \mathrm{d} r=\frac{8 \pi}{f^{3}} \int_{\tau_{0}}^{\frac{R f}{2}} S^{2} \lambda(S) \mathrm{d} S \\
& \text { for all } \quad f \in f_{o}+\mathbb{P}^{\times} .
\end{aligned}
$$

This formula can be used to express the function $\lambda$ in terms of the function $Q$. In fact, we have

$$
\lambda(S)=\frac{1}{4 \pi R S^{2}} \bar{Q}^{\bullet}\left(\frac{2 S}{R}\right) \quad \text { for all } \quad S \in \tau_{o}+\mathbb{P}^{\times},
$$

where $\bar{Q}: f_{o}+\mathbb{P}^{\times} \rightarrow \mathbb{P}$ is defined by

$$
\bar{Q}(f):=f^{3} Q(f) \quad \text { for all } \quad f \in f_{o}+\mathbb{P}^{\times} .
$$


The function $Q$ may be amenable to determination by experiment. One can then use (24) and (25) to calculate the rate of shear function $\lambda$ and hence the shear stress function $\tau$.

\subsubsection{Bingham materials}

If the semi-liquid is a Bingham material then $v_{p}$ can be calculated explicitly with (4) to yield

$$
v_{p}=\frac{f}{4 \eta_{o}}(R-P)^{2} .
$$

Also, (23) can be evaluated with the following result:

$$
\begin{aligned}
& Q(f)=\frac{\pi R^{4} f}{8 \eta_{o}}\left(1-\frac{4}{3}\left(\frac{2 \tau_{o}}{f R}\right)+\frac{1}{3}\left(\frac{2 \tau_{o}}{f R}\right)^{4}\right) \\
& \text { for all } \quad f \in f_{o}+\mathbb{P}^{\times} .
\end{aligned}
$$

This formula is known, in the literature of rheology, as the Buckingham-Reiner equation.

\subsection{Flow Between Concentric Cylinders, General Results}

We consider a steady flow of a semi-liquid between two infinite concentric cylinders of radii $R_{1}$ and $R_{2}$, with $R_{1}<R_{2}$. They may rotate about their common axis with angular velocities $\Omega_{1}$ and $\Omega_{2}$ and may slide in the direction of their axes with velocities $U_{1}$ and $U_{2}$, respectively. Also, there may be a driving force in the axial direction exerted on the material directly, not to be confused with the forces acting on the cylinders needed to produce the sliding.

Choose a cylindrical coordinate system in such a way that the z-axis coincides with the axes of the cylinders and choose the positive $z$-direction so that the specific driving force $f$ is positive (strictly positive or zero). The velocities $U_{1}$ and $U_{2}$ are positive if the cylinders are sliding in the positive z-direction and negative otherwise. It is assumed the velocity field has the form

$$
v^{r}=0, \quad v^{z}=u(r), \quad v^{\theta}=\omega(r)
$$

relative to these cylindrical coordinates. If both $u$ and $\omega$ are non-zero, the flow is helical in the sense that the stream lines are helices. If $u$ is zero but $\omega$ is not, the streamlines are circles. If $\omega$ is zero but $u$ is not, the streamlines are straight lines.

Assuming that the material adheres to the cylinders, we have the boundary conditions

$$
\begin{array}{ll}
u\left(R_{1}\right)=U_{1}, & u\left(R_{2}\right)=U_{2}, \\
\omega\left(R_{1}\right)=\Omega_{1}, & \omega\left(R_{2}\right)=\Omega_{2} .
\end{array}
$$

The analysis in 3) (p.37-41) remains valid. The shear stress at the distance $r$ from the axis is given by (16.8) of 3), i.e., by the restriction to $\left[R_{1}, R_{2}\right]$ of function $S: \mathbb{P}^{\times} \rightarrow \mathbb{P}$ defined by

$$
\begin{aligned}
S(r) & =\left[\left(\frac{M}{2 \pi r^{2}}\right)^{2}+\left(\frac{b}{r}-\frac{r f}{2}\right)^{2}\right]^{\frac{1}{2}} \\
& =\left[\left(\frac{M}{2 \pi r^{2}}\right)^{2}+\frac{b^{2}}{r^{2}}-b f+\frac{r^{2} f^{2}}{4}\right]^{\frac{1}{2}},
\end{aligned}
$$

where $M$ is the torque per unit height required to maintain the relative rotation of the cylinders. The constant $b$ is to be determined later.

One can solve the dynamical equations (see (16.10) and (16.11) of 3)) with the above boundary conditions to obtain:

$$
\begin{aligned}
& u(r)=\int_{R_{1}}^{r}\left(\frac{b}{\xi}-\frac{\xi f}{2}\right) \frac{\lambda(S(\xi))}{S(\xi)} d \xi+U_{1}, \\
& \omega(r)=\frac{M}{2 \pi} \int_{R_{1}}^{r} \frac{\lambda(S(\xi))}{\xi^{3} S(\xi)} d \xi+\Omega_{1},
\end{aligned}
$$

for $r \in\left[R_{1}, R_{2}\right]$, so that

$$
\begin{aligned}
& U:=U_{2}-U_{1}=\int_{R_{1}}^{R_{2}}\left(\frac{b}{\xi}-\frac{\xi f}{2}\right) \frac{\lambda(S(\xi))}{S(\xi)} d \xi, \\
& \Omega:=\Omega_{2}-\Omega_{1}=\frac{M}{2 \pi} \int_{R_{1}}^{R_{2}} \frac{\lambda(S(\xi))}{\xi^{3} S(\xi)} d \xi .
\end{aligned}
$$

$U$ is the relative velocity of the sliding cylinders in the $z$-direction, and $\Omega$ is the relative angular velocity of the rotating cylinders.

We say that there is a plug if both $u$ and $\omega$ have constant values in some subinterval of $\left[R_{1}, R_{2}\right]$. If the right end of this interval is $R_{2}$ we say that the plug adheres to the outer cylinder, if the left end is $R_{1}$ we say that the plug adheres to the inner cylinder. Otherwise, we say that the plug is internal.

We now make some case distinctions.

Case 1: $f>0$ and $M \neq 0$

It is clear from (31) that Rng $S \subset \mathbb{P}^{\times}$and that the function $S$ is difierentiable. An easy calculation shows that

$$
S^{\bullet}(r)=-S(r)^{-1}\left(\frac{M^{2}}{2 \pi^{2} r^{5}}+\frac{b^{2}}{r^{3}}-\frac{r f^{2}}{4}\right) .
$$

It is easily seen that $S^{\bullet}$ only has one root. Also, by (31), we have

$$
\lim _{r \rightarrow 0} S(r)=\infty=\lim _{r \rightarrow \infty} S(r) .
$$

Therefore, the graph of $S$ has the form shown in Fig. 2. The function $S$ has a minimum $S_{m}$ which is obtained at $r_{m}$, so that $S_{m}=S\left(r_{m}\right)$. Since $S^{\bullet}\left(r_{m}\right)=0$, it follows from (36) that

$$
0=\frac{M^{2}}{2 \pi^{2} r_{m}^{5}}+\frac{b^{2}}{r_{m}^{3}}-\frac{r_{m} f^{2}}{4} .
$$

If $\tau_{o}>S_{m}$, then there are $P_{1}, P_{2} \in \mathbb{P}^{\times}$, with $P_{1}<r_{m}<R_{2}$, such 
that $S\left(P_{1}\right)=S\left(P_{2}\right)=\tau_{o}$ (see Fig. 2). Since $M \neq 0$, it follows from (37) that $\frac{4 b^{2}}{f^{2}}<r_{m}^{4}$ and hence

$$
\frac{4 b^{2}}{f^{2}}<r^{4} \quad \text { for all } \quad r \in r_{m}+\mathbb{P} .
$$

Case 2: $f>0, M=0$ and $b>0$

In this case, (31) reduces to

$$
S(r)=\left|\frac{b}{r}-\frac{r f}{2}\right|
$$

Then $S$ has the minimum $S_{m}=0$ at $r_{m}$, so that $b=\frac{r_{m}^{2} f}{2}$. Then, by (39)

$$
S(r)=\frac{f}{2 r}\left|r_{m}^{2}-r^{2}\right|
$$

Hence,

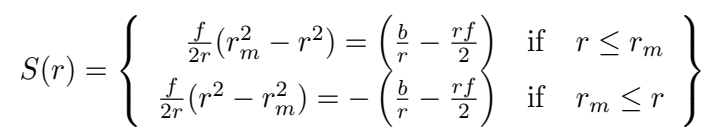

and

$$
S^{\bullet}(r)=\left\{\begin{array}{rll}
-\frac{f}{2} \frac{r^{2}+r_{m}^{2}}{r^{2}} & \text { if } & r<r_{m} \\
\frac{f}{2} \frac{r^{2}+r_{m}^{2}}{r^{2}} & \text { if } & r_{m}<r
\end{array}\right\} .
$$

The left and right limits of $S^{\bullet}$ at $r_{m}$ are $-f$ and $f$, respectively. Also, $S$ has the property that $\lim _{r \rightarrow 0} S(r)=\infty=\lim _{r \rightarrow \infty} S(r)$ and so the graph of $S$ has the form shown in Fig. 3. Since $\tau_{o}>0$, there are $P_{1}, P_{2} \in \mathbb{P}^{\times}$, with $P_{1}<r_{m}<P_{2}$, such that $S\left(P_{1}\right)=S\left(P_{2}\right)=\tau_{o}$, i.e., by $(41)$

$$
\frac{f}{2 P_{1}}\left(r_{m}^{2}-P_{1}^{2}\right)=\tau_{o}=\frac{f}{2 P_{2}}\left(P_{2}^{2}-r_{m}^{2}\right) .
$$

A simple calculation from (43) shows that

$$
\tau_{o}=\frac{f}{2}\left(P_{2}-P_{1}\right) \text { and } r_{m}^{2}=P_{1} P_{2}
$$

Assume now that $R_{1}<r_{m}<R_{2}$. Then, by (41)

$$
\frac{b}{r}-\frac{r f}{2}:=\left\{\begin{array}{rll}
S(r) & \text { if } & r \in\left[R_{1}, r_{m}\right] \\
-S(r) & \text { if } & r \in\left[r_{m}, R_{2}\right]
\end{array}\right\} .
$$

Hence, by (34)

$$
U=\int_{R_{1}}^{r_{m}} \lambda(S(r)) d r-\int_{r_{m}}^{R_{2}} \lambda(S(r)) d r .
$$

Case 3: $f=0$

In this case, (31) reduces to

$$
S(r)=\left(\frac{M^{2}}{4 \pi^{2} r^{4}}+\frac{b^{2}}{r^{2}}\right)^{1 / 2}
$$

It is clear that the function $S$ is strictly antitone and that $\lim _{r \rightarrow 0}$ $S(r)=\infty$ and $\lim _{r \rightarrow \infty} S(r)=0$. Therefore, the graph of $S$ has the form shown in Fig. 4. Since $\tau_{o}>0$, there is a exactly one $P \in \mathrm{IP}^{\times}$ such that $S(P)=\tau_{o}$. Hence, by (47)

$$
b^{2}=P^{2} \tau_{o}^{2}-\frac{M^{2}}{4 \pi^{2} P^{2}}
$$

The following are two results of a general nature.

Theorem 1: Assume that $f>0$. If there is a plug attached to the inner cylinder, then $U<0$. Hence, if $U \geq 0$, any possible plug must be internal or attached to the outer cylinder.

Proof: Assume there is a plug attached to the inner cylinder. Then we must have $\tau_{o}>S_{m}$ and $P_{1} \leq R_{1}<P_{2}<R_{2}$, where $P_{1}$ and $P_{2}$ are determined as in Case 1 or Case 2 above, so that $S(r) \leq \tau_{0}$, and hence $\lambda(S(r))=0$, for all $r \in\left[R_{1}, P_{2}\right]$. It follows

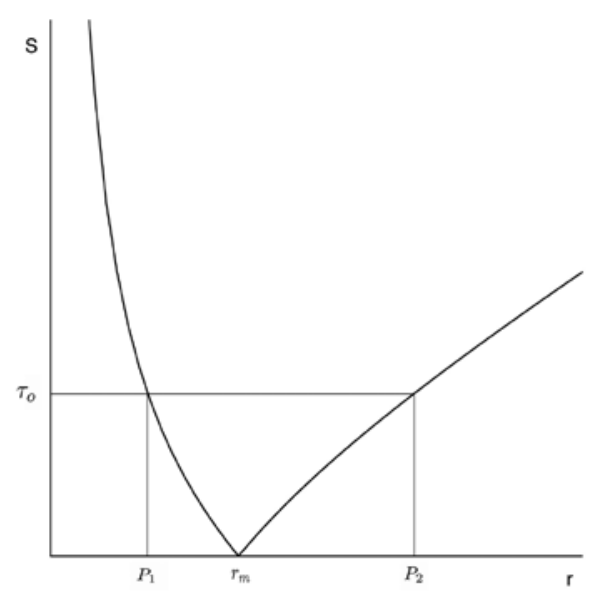

Fig. 3. Shear stress as a function of radius, Case 2 .

Fig. 2. Shear stress as a function of radius, Case 1 . 
from (32) and (33) that $u(r)=U_{1}$ and $\omega(r)=\Omega_{1}$ for all $r \in$ $\left[R_{1}, P_{2}\right]$. From (34) one obtains that

$$
U=\int_{P_{2}}^{R_{2}}\left(\frac{b}{\xi}-\frac{\xi f}{2}\right) \frac{\lambda(S(\xi))}{S(\xi)} d \xi .
$$

Now, if $b \leq 0$, then the integrand of (49) is strictly negative and hence $U<0$. If $b>0$, it follows from (38) when $M \neq 0$ and from (45) when $M=0$ that $\frac{b}{r}-\frac{r f}{2} \leq 0$ for all $r \in r_{m}+\mathbb{P}$. Since $r_{m}<P_{2}$, it follows that the integrand in (49) is still strictly negative and hence $U<0$.

Theorem 2: Assume that $f=0$. Then any possible plug must be attached to the outer cylinder.

Proof: Let $P$ be determined as in Case 3 above. If there is a plug, we must have $R_{1}<P<R_{2}$. We have that $S(r) \leq \tau_{o}$, and hence $\lambda(S(r))=0$, for all $r \in P+\mathbb{P}^{\times}$. It follows from (32) and (33) that $u(r)=U_{2}$ and $\omega(r)=\Omega_{2}$ for all $r \in\left[P, R_{2}\right]$. This means that the plug adheres to the outer cylinder.

\subsection{Couette Flow}

Couette flow is a flow between concentric cylinders in which the boundary condition (29) is modified so that

$$
U_{1}=U_{2}=0 \quad \text { and } \quad f=0 .
$$

We are in Case 3 of Sect. 4.3. It follows from (34) that $b=0$. The formulas (31), (32) and (33) then reduce to

$$
\begin{aligned}
& S(r)=\frac{M}{2 \pi r^{2}}, \quad u(r)=0 \\
& \text { and } \omega(r)=\int_{R_{1}}^{r} \frac{1}{\xi} \lambda\left(\frac{M}{2 \pi \xi^{2}}\right) d \xi+\Omega_{1} .
\end{aligned}
$$

It follows from (48) that

$$
P:=\sqrt{\frac{M}{2 \pi \tau_{o}}} .
$$

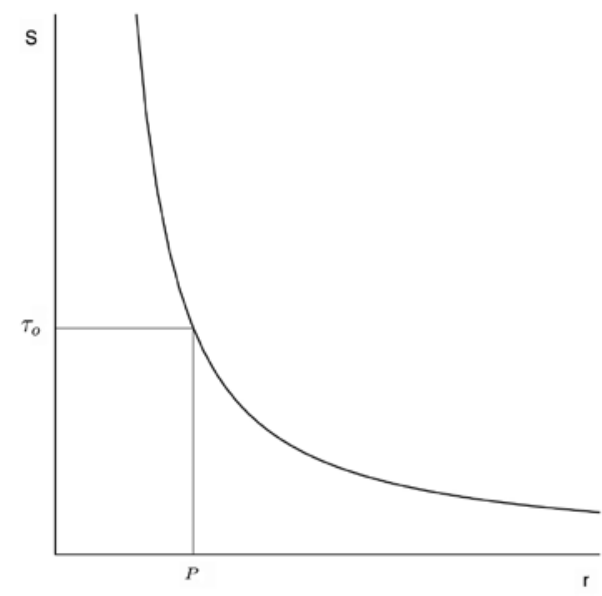

Fig. 4. Shear stress as a function of radius, Case 3.
It is clear from (52) and (51) that there is a plug if and only if $R_{1}<P<R_{2}$. Defining

$$
M_{1}:=2 \pi R_{1}^{2} \tau_{o}, \quad M_{2}:=2 \pi R_{2}^{2} \tau_{o},
$$

this means that there is a plug if and only if $M_{1}<M<M_{2}$.

\subsubsection{Results}

If $M \leq M_{1}$, and hence $P \leq R_{1}$, then the integrand in (51) is always zero and hence $\omega(r)=\Omega_{1}=\Omega_{2}$ for all $r \in\left[R_{1}, R_{2}\right]$. Thus, the material rotates rigidly at a constant angular velocity.

If $M \geq M_{2}$, and hence $P \geq R_{2}$, then the integrand in (51) is never zero so there are no plugs.

If $M_{1}<M<M_{2}$, and hence $R_{1}<P<R_{2}$, then the integrand in (51) $)_{3}$ is zero for $r \in\left[P, R_{2}\right]$ and hence corresponds to a plug attached to the outer cylinder. Using $(30)_{2}$ it is easy to see that the angular velocity of the plug is $\Omega_{2}$.

It follows that (35) reduces to

$$
\Omega=\frac{M}{2 \pi} \int_{R_{1}}^{P} \frac{\lambda(S(\xi))}{\xi^{3} S(\xi)} d \xi
$$

Let $\hat{\Omega}:\left[M_{1}, M_{2}\right] \rightarrow \mathbb{P}$ describe the angular velocity difierence $\Omega$ as a function of the torque per unit height needed to produce the flow. In view of (54), this means that

$$
\hat{\Omega}(M)=\int_{\tau_{o}}^{\frac{M}{2 \pi R_{1}{ }^{2}}} \frac{\lambda(S)}{2 S} \mathrm{~d} S \quad \text { for all } \quad M \in\left[M_{1}, M_{2}\right] .
$$

This formula can be used to express the function $\lambda$ in terms of the function $\hat{\Omega}$. In fact, we have

$$
\begin{aligned}
& \lambda(S)=4 \pi R_{1}{ }^{2} S \hat{\Omega}^{\bullet}\left(2 \pi R_{1}{ }^{2} S\right) \\
& \text { for all } S \in\left[\tau_{o},\left(\frac{R_{2}}{R_{1}}\right)^{2} \tau_{o}\right] .
\end{aligned}
$$

The function $\hat{\Omega}$ may be amenable to determination by experiment. One can then use (56) to calculate the rate of shear function $\lambda$ and hence the shear stress function $\tau$.

\subsubsection{Bingham materials}

For such materials, (55) can be made explicit with the result

$$
\hat{\Omega}(M)=\frac{\tau_{o}}{2 \eta_{o}}\left(\frac{M}{2 \pi \tau_{o} R_{1}^{2}}-\log \frac{M}{2 \pi \tau_{o} R_{1}^{2}}-1\right) .
$$

\subsection{Concentric Pipe Flow}

In this section we consider a steady flow between concentric cylinders in which the boundary conditions (29) and (30) are of the form

$$
U_{1}=U_{2}=\Omega_{1}=\Omega_{2}=0 .
$$


It follows from (35) that $M=0$ and hence $\omega(r)=0$ for all $r$ $\in\left[R_{1}, R_{2}\right]$. Of course, if $f=0$ there is no flow. Hence we may assume that $f>0$.

The equation (34) reduces to

$$
0=\int_{R_{1}}^{R_{2}}\left(\frac{b}{r}-\frac{r f}{2}\right) \frac{\lambda(S(r))}{S(r)} d r .
$$

If $b \leq 0$ then the integrand in (59) is strictly negative unless $S(r) \leq \tau_{o}$ for all $r \in\left[R_{1}, R_{2}\right]$, which means, by (2) and (32), that there is no flow.

Therefore, we now assume that $b>0$, and so we are in Case 2 of Sect. 4.3. If $R_{1}<r_{m}<R_{2}$ fails to hold, it follows from (41) that the integrand in (59) is either strictly positive or strictly negative unless $S(r) \leq \tau_{o}$ for all $r \in\left[R_{1}, R_{2}\right]$, which means, by (2) and (32) again, that there is no flow.

Therefore we now assume that $R_{1}<r_{m}<R_{2}$, and hence, by (46), that

$$
\int_{R_{1}}^{r_{m}} \lambda(S(r)) d r=\int_{r_{m}}^{R_{2}} \lambda(S(r)) d r .
$$

Recall that $S(r) \leq \tau_{o}$ for all $r \in\left[P_{1}, P_{2}\right]$. Thus, there can be a flow only if

$$
R_{1}<P_{1}<r_{m}<P_{2}<R_{2}
$$

We now assume that (61) holds. By (44) we then must have

$$
\frac{2 \tau_{o}}{f}=P_{2}-P_{1}<R_{2}-R_{1}
$$

There is an internal plug described by the interval $\left[P_{1}, P_{2}\right]$.

By (32), (58), and (41) we have

$$
u(r)=\left\{\begin{array}{lll}
\int_{R_{1}}^{r} \lambda(S(\xi)) d \xi & \text { if } & r \in\left[R_{1}, P_{1}\right] \\
\int_{r}^{R_{2}} \lambda(S(\xi)) d \xi & \text { if } & r \in\left[P_{2}, R_{2}\right]
\end{array}\right\} .
$$

The speed of the plug is given by

$$
v_{p}=u\left(P_{1}\right)=u\left(P_{2}\right)=\int_{R_{1}}^{P_{1}} \lambda(S(r)) d r=\int_{P_{2}}^{R_{2}} \lambda(S(r)) d r .(64)
$$

In view of (41), it follows that

$$
v_{p}=\int_{R_{1}}^{P_{1}} \lambda\left(\frac{f}{2 r}\left(r_{m}^{2}-r^{2}\right)\right) d r=\int_{P_{2}}^{R_{2}} \lambda\left(\frac{f}{2 r}\left(r^{2}-r_{m}^{2}\right)\right) d r .
$$

The equations (44) and (65) can be used to determine $r_{m}, P_{1}$ and $P_{2}$.

\subsubsection{Results}

If $f \leq \frac{2 \tau_{o}}{R_{2}-R_{1}}$ then there is no flow.

If $f>\frac{2 \tau_{o}}{R_{2}-R_{1}}$ then there is flow and the internal plug is described by the subinterval $\left[P_{1}, P_{2}\right]$ of $\left[R_{1}, R_{2}\right]$.
The equations (44) and (65) can be used to determine $r_{m}, P_{1}$ and $P_{2}$.

The volume discharge $Q(f)$ as a function of the specific driving force $f$ for this flow is given by (18.7) in 3). In this case, it reduces to

$$
\begin{aligned}
Q(f)=\pi\left(\int_{R_{1}}^{P_{1}} r^{2} \lambda\left(\frac{f}{2 r}\left(r_{m}^{2}-r^{2}\right)\right) d r\right. \\
+\int_{P_{2}}^{R_{2}} r^{2} \lambda\left(\frac{f}{2 r}\left(r^{2}-r_{m}^{2}\right) d r\right) .
\end{aligned}
$$

Remark: It can be shown that in the limit as $R_{1}$ goes to infinity, while $R_{2}-R_{1}$ and $f$ are kept fixed, the flow approaches that of a channel flow.

\subsubsection{Bingham materials}

When the semi-liquid is a Bingham material, the rate of shear function $\lambda$ is given by (4) and one can evaluate the integrals in (65) explicitly with the result

$$
\begin{aligned}
& v_{p}=\frac{f}{4 \eta_{o}}\left(2 r_{m}^{2} \log \left(\frac{P_{1}}{R_{1}}\right)-\left(P_{1}^{2}-R_{1}^{2}\right)-\frac{4 \tau_{o}}{f}\left(P_{1}-R_{1}\right)\right) \\
& =\frac{f}{4 \eta_{o}}\left(-2 r_{m}^{2} \log \left(\frac{R_{2}}{P_{2}}\right)+\left(R_{2}^{2}-P_{2}^{2}\right)-\frac{4 \tau_{o}}{f}\left(R_{2}-P_{2}\right)\right) .
\end{aligned}
$$

Using $(44)_{2}$, it follows from (67) that

$$
\begin{aligned}
2 P_{1} P_{2} \log \left(\frac{R_{2} P_{1}}{R_{1} P_{2}}\right)= & \left(R_{2}^{2}-R_{1}^{2}+P_{1}^{2}-P_{2}^{2}\right) \\
& +\frac{4 \tau_{o}}{f}\left(P_{1}+P_{2}-R_{2}-R_{1}\right) .
\end{aligned}
$$

Using (44) $)_{1}$, we can substitute $P_{2}=P_{1}+\frac{2 \tau_{o}}{f}$ into (68) to obtain an equation for $P_{1}$ that can be solved numerically and then also calculate $P_{2}$.

The formula (65) can be evaluated explicitly to yield, with the help of (44) $)_{2}$,

$$
\begin{aligned}
& Q(f)=\frac{\pi f}{\eta_{o}}\left[\frac{1}{4} P_{1} P_{2}\left(P_{1}^{2}+P_{2}^{2}-R_{2}^{2}-R_{1}^{2}\right)\right. \\
& \left.-\frac{1}{8}\left(P_{1}^{4}+P_{2}^{4}-R_{2}^{4}-R_{1}^{4}\right)-\frac{\tau_{o}}{3 f}\left(P_{1}^{3}-P_{2}^{3}+R_{2}^{3}-R_{1}^{3}\right)\right] .
\end{aligned}
$$

\subsection{Cylindrical Shearing}

Here we look at the concentric cylinder case when $f=M=0$ but $U \neq 0$. We are in Case 3 of Sect. 4.3. and (47) and (48) reduce to

$$
S(r)=\frac{P \tau_{0}}{r} .
$$

Therefore, (34) reduces to

$$
U=\hat{U}(P)=\int_{R_{1}}^{R_{2}} \lambda\left(\frac{P \tau_{o}}{r}\right) d r
$$


It is clear that the function $\hat{U}: \mathbb{P} \rightarrow \mathbb{P}$ is strictly isotone and surjective. Thus, for any given $U$, there is exactly one $P$ such that (70) holds.

\subsubsection{Results}

If $U \geq \hat{U}\left(R_{2}\right)$ then we must have $P \geq R_{2}$, and there is no plug.

If $P<R_{2}$ then there is a plug attached to the outer cylinder. It is described by the interval $\left[P, R_{2}\right]$ and (71) reduces to

$$
U=\hat{U}(P)=\int_{R_{1}}^{P} \lambda\left(\frac{P \tau_{o}}{r}\right) d r .
$$

The force per unit length $F$ necessary to maintain the speed of the outer cylinder is given by

$$
F=2 \pi S\left(R_{2}\right)=2 \pi \frac{P \tau_{o}}{R_{2}} .
$$

Thus, if $F$ is given, (73) can be used to determine $P$ and then (72) to determine $U$.

Remark: It can be shown that in the limit as $R_{1}$ goes to infinity, while $R_{2}-R_{1}$ and $U$ are kept fixed, the inequality $U \geq \hat{U}\left(R_{2}\right)$ always holds and so the flow approaches that of simple shearing.

\subsubsection{Bingham materials}

In this case (72) can be evaluated explicitly with the result

$$
U=\hat{U}(P)=\frac{1}{\eta_{o}}\left[P \tau_{o} \log \left(\frac{P}{R_{1}}\right)+\tau_{o}\left(R_{1}-P\right)\right] .
$$

\section{CONCLUSIONS}

In this paper we have found under what conditions simple semi-fluids admit plugs in viscometric flows. We have determined that in simple shearing, cone and plate and torsional flow there are no plugs. However, in these flows a critical force or torque is required to obtain a flow (see Sects. 3.1.1, 3.2.1 and 3.3.1). In channel, Poiseuille, Couette and concentric pipe flow it was found that in certain situations plugs can occur (see Sects. 4.1.1, 4.2.1, 4.4.1, 4.5.1 and 4.6.1).
In the cases where plugs can occur, and when the concept makes sense, we have given formulae for the volume discharge (see equations (17), (23), (27) and (66)), which can be used to find the shear-stress function of the material, and the speed of the plug (see equations (15), (22), (26) and (65)). Also, we have obtained explicit formulae for the volume discharge and the speed of the plug in the case of a Bingham material (see Sects. 4.1.2, 4.2.2, 4.4.2, 4.5.2 and 4.6.2).

\section{Acknowledgment}

We are grateful to Guy Berry for helpful remarks and proofreading.

\section{REFERENCES}

1) Noll W, A mathematical theory of the mechanical behavior of continuous media, Archive for Rational Mechanics and Analysis 2, 197-226 (1958).

2) Truesdell C, Noll W, The Non-Linear Field Theories of Mechanics, Encyclopedia of Physics, Vol. III/3, 602 pages. Springer-Verlag, 1965. Second Edition, 1992. Translation into Chinese, 2000. Third Edition, 2004.

3) Coleman BD, Markovitz H, Noll W, Viscometric Flows of Non-Newtonian Fluids, Theory and Experiment, 130 pages, Springer Tracts in Natural Philosophy 5, Springer-Verlag, 1966.

4) Noll W, A new mathematical theory of simple materials, Archive for Rational Mechanics and Analysis 48, 1-50 (1972).

5) Noll W, Five Contributions to Natural Philosophy, 73 pages, 2004.

Part 4: The Theory of Simple Semi-Liquids, a Conceptual Framework for Rheology.

Part 5: Nematic Semi-Liquids.

Published on the website www.math.cmu.edu/ wn0g/noll.

6) Cruz DOA, Pinho FT, Skewed Poiseuille-Couette flows of SPPT fluids in concentric annuli and channels, Journal of Non-Newtonian Fluid Mechanics, 121, (2004).

7) Noll W, Finite-Dimensional Spaces, Algebra, Geometry, and Analysis, Vol.I, 393 pages. Martinus Nijhoff Publishers, 1987. A corrected version (2005)is published on the website www. math.cmu.edu/ wn0g/noll. 